\title{
Rejuvenation of Aged Hematopoietic Stem Cells
}

\section{Yariv Greenshpan ${ }^{1-3}$ and Roi Gazit ${ }^{1-3^{*}}$}

${ }^{1}$ The Shraga Segal Department for Microbiology, Immunology and Genetics, Faculty of Health Science, Ben-Gurion University of the Negev, Beer Sheva 84105, Israel

${ }^{2}$ The National Institute for Biotechnology in the Negev, Ben-Gurion University of the Negev, Beer Sheva 84105, Israel

${ }^{3}$ Regenerative Medicine and Stem Cell Research Center, Ben-Gurion University of the Negev, Beer Sheva 84105, Israel

Due to advances in medicine and public health programs we are living longer than ever. Aging affects many biological processes, among them is the immune system ability to respond to vaccinations and fight infections, which declines as we age $[1,2]$. Strikingly, the aged population is extremely susceptible to influenza and $80-90 \%$ of influenza related mortalities occurs in patients of 65 years old or older [3]. Elderly may also suffers from autoimmunity [4], another indication for irregularities in the aged immune response. Sub-clinical anemia and increased frequencies of specific leukemia types further highlight the health impact of the hematopoietic system [2]. It is clear that there is a need to improve and rejuvenate an individual's aged immune response but is it feasible and if so how?

Hematopoietic stem cells (HSCs) are the continuous source of the various lymphocytes and myeloid cells from early development and through aging. HSC's ability to self-renew and sustain multipotency underlay their potential as Adult Stem Cells, and requires adequate regulation of proliferation [2]. Numerous signals, intrinsic and extrinsic, are in place to maintain the balance between proliferation and quiescence - the state in which HSCs do not divide for prolonged time without losing their potential [5]. Adequate quiescence is thought to have an essential role in the life-long potential of HSCs, and in their aging. Both intrinsic and extrinsic mechanisms are known to regulate HSCs, for instance overexpressing of Hox genes can increase HSC's self-renewal [6], and changes of the bone-marrow niche can affect the number and location of HSCs [7]. One of the most remarkable properties of HSCs is the shier potential for self-renewal that they hold, which greatly superposes ones lifespan as demonstrated using serial transplantation assay [8]. Importantly, HSCs function as individual cells, but are making a population of cells which differs in terms of self-renewal potential; when trying to assess an HSC lifespan in a clonal manner it has been demonstrated that certain clones in the HSC population can out live the original host but not all HSCs do so [9]. If HSCs have evidently the potential to generate normal hematopoiesis for longer than our lifespan it should be possible to make them work better within our life time. This raises the question: how can we treat our own HSCs to rejuvenate the immune system?

The term "aged stem cell" is somewhat paradoxical. One of the fundamental properties of a stem cell, is its ability to make more of itself while keeping original potency [10]. Even so, to date substantial evidences have accumulated for aged stem cells and several models have been proposed for the aging of HSCs, we present our simplified model in Figure 1, including the major features of self-renewal potency, differentiation potentials, and population vs. single-cell activities. The effect of aged stem cells on the corresponding tissue or organ varies much according to homeostasis rates of exchanging old cells with new ones. The aged immune response is well documented and sometimes referred to as "immuno-senescence". It is considered to be a gradual process which leads to a decrease in the ability to effectively respond to infection and to create long term immune memory [11]. The HSCs of both aged mice and human show an increase tendency to differentiate to the myeloid lineage rather than the lymphoid lineage $[12,13]$, a phenomenon referred to as the myeloid bias. Even earlier studies had pointed that while HSC's population expands substantially with age, not all retain their balanced-differentiation -- but they rather demonstrate a strict myeloid-bias while retaining self-renewal potency [14]. Multiple studies had further elucidated potential mechanisms, which are beyond the scope of this paper and are better presented in a review [15]. The result of lineage- bias is a reduced number of new B-cells and naïve T-cells $[16,17]$. The ration of myeloid-lymphoid bias is not the only parameter affected in the aged immune response. It appears that aging impairs the T-cells response to dendritic cells [18] and a decrease in antibody affinity in the affinity maturation process [19]. Clearly, physiological processes such as thymic involution may have independent impact, but might yet be connected with the bone-marrow hematopoiesis. All this combined leads to a dramatic decrease in the efficiency of the immune response as we age. From a rejuvenation point of view, understanding the pillars of immune-aging provides potential targets when looking to avoid immune-aging. Yet, it is an open question whether we may reverse the situation after immune activity already declined?

Rejuvenation aims specifically to reverse aged-phenotype back into young state, in contrast with other approaches that aims to delay aging. Several anti-aging approaches are fairly known, perhaps most famous is the restriction of food which is better known as "caloric restriction". While reducing food intake achieved significant delay of aging in many lower-organisms, its impact on mammals and humans is less simple, and importantly face the edge of malnutrition that tragically impairs the immune system activity [20,21]. Nevertheless, one recent study suggested that an acute severe food limitation, as prolonged fasting, may actually reverse aged state in mouse [22], with a focus on IGF-1 pathway. Cdc42 was also reported to have a major role on HSCs aging, with potential rejuvenation for its targeting [23]. Immune rejuvenation of effector-cells function was demonstrated using cytokines, hormones and vitamins - but such studies mostly focused on differentiated rather than stem cells. Recently, the ablation of senescent cells using a novel compound reported rejuvenation of both HSCs and muscle-stemcells in normal aged mouse [24], further emphasizing that the potency of adult stem cells persist through aging. Taken together, multiple approaches suggest that we can not only postpone aging, but also revert it as rejuvenation literally means. Therefore, Next question is what are the molecular mechanisms by which HSCs can be directed from aged state into young functionality?

*Corresponding author: Roi Gazit, The Shraga Segal Department for Microbiology, Immunology and Genetics, Faculty of Health Science, BenGurion University of the Negev, Beer Sheva, Israel, Tel: 9728 6477272; E-mail: gazitroi@bgu.ac.il

Received August 31, 2016; Accepted September 01, 2016; Published September 08,2016

Citation: Greenshpan Y, Gazit R (2016) Rejuvenation of Aged Hematopoietic Stem Cells. J Clin Med Genomics 4: 142. doi: 10.4172/2472-128X.1000142

Copyright: @ 2016 Greenshpan Y, et al. This is an open-access article distributed under the terms of the Creative Commons Attribution License, which permits unrestricted use, distribution, and reproduction in any medium, provided the original author and source are credited. 


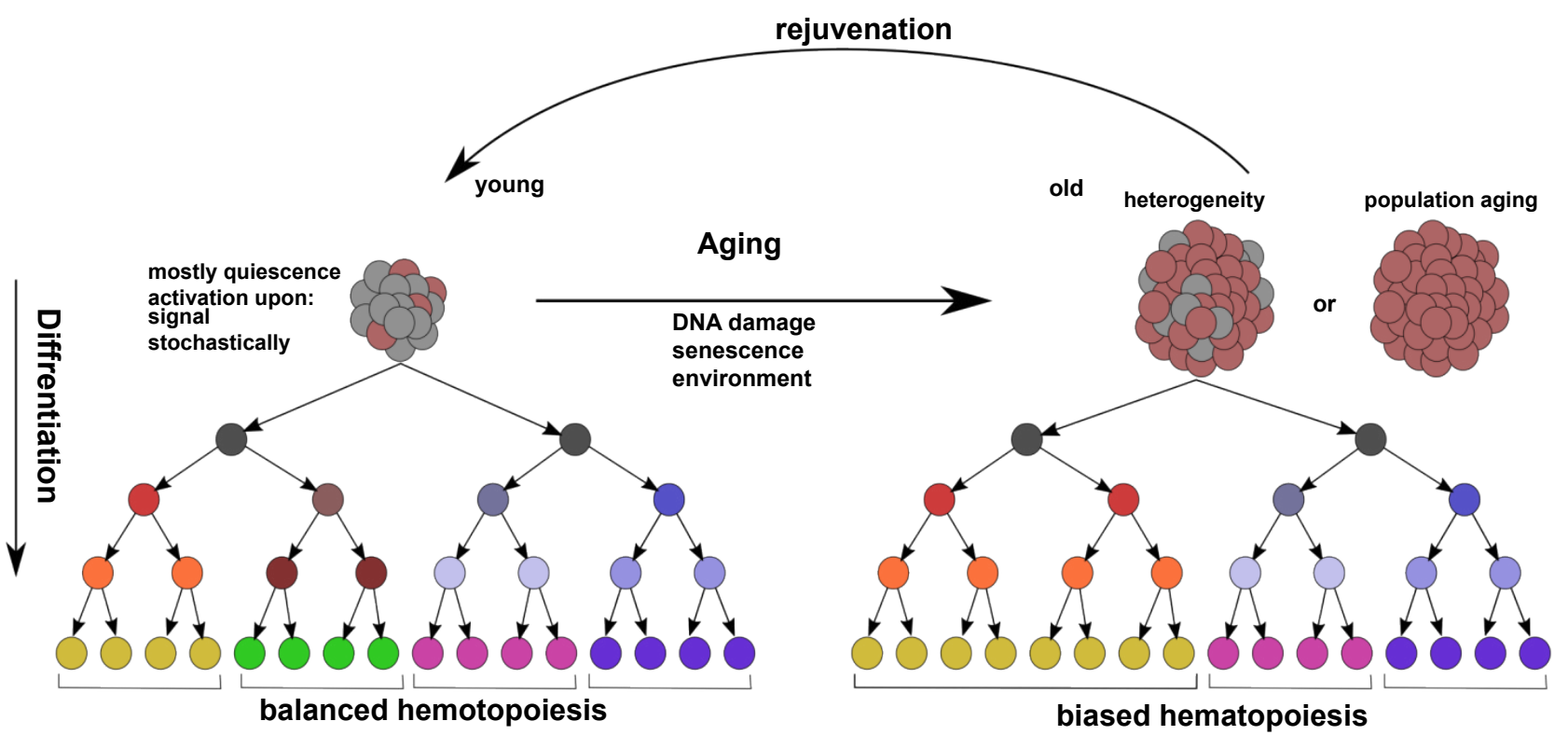

Figure 1: Schematic model for HSCs aging and rejuvenation, representing the common concepts regarding normal hematopoiesis and the outcomes of aged HSCs with emphasis to myeloid bias and single-cell vs. population changes. Rejuvenation is including cell-intrinsic as well as extrinsic modifications.

HSCs generate all types of immune and blood cells, letting a rational for having a feedback signaling from the mature effector cells to sustain homeostasis. Indeed, it was reported that bleeding induced HSC's activation that could be blocked with erythrocytes but not with leukocytes [25]. However, this might not correlate with lineage-bias, and aging of the immune system involves accumulation of lymphocytes not erythrocytes. The chronic deficiency of B-cells had been found to specifically prevent their aging [26], suggesting that immuno-senescence involves feedback mechanisms. This was elegantly demonstrated using specific depletion of B-cells in aged mice, which induced not only the generation of new B-cells but also their functional response to vaccine and the rejuvenation of the more primitive stem- and progenitors compartments [27]. These studies demonstrate that rejuvenation of the immune system, from stem cells through progenitors and effectors functions can be achieved via the regulation of cellular homeostasis. Identification of the mechanisms by which homeostasis is sustained, and by which stress-hematopoiesis is induced, are therefore essential for the development of new rejuvenation treatments.

HSCs are formed during mid-gestation, and persist throughout life as individual clones making a population, but keeping each cell separately. Individual HSC are known to differ functionally, in part due to their inherent history and in part due to technical and stochastic variability of experimental procedures. Importantly, several studies reported the isolation of distinct HSC's subsets. It has also been suggested that the myeloid bias is not due to a change in the differentiation potential of single HSCs but rather by a gradual selection process leading to a preference of a certain subtype over the other [28]. Later studies prospectively isolated such HSC-subsets that differ in their myleo-lymphoid differentiation bias [29-31]. Nevertheless, even the most purified immuno-phenotype HSCs still differ individually. Therefore, it was suggested that aging might consist of changing frequencies of different HSC's clones within the population, rather than individual change in each and every cell. If this scenario is true, then rejuvenation may aim not to change cells but rather to change the composition of stem cell's population. However, there are additional studies that demonstrate further changes, elegantly reviewed recently [15], suggesting that in parallel to the shift in clonal-composition there is an intrinsic aging in the majority of HSCs, including DNA-damage accumulation and epigenetic changes over time. We may note that DNA-damage must be fixed during cell-division, and epigenetics marks are possible to remodeling. However, HSCs of old mice were reported to persist their "aged" function and profiles through transplantation, and erase it only with the extreme reprogramming to pluripotency [32]. This suggests that virtually all known aging mechanisms might be reversed, and there is no known hard limitation over the potency of aged HSCs, perhaps endogenously and not through transplantation. It is yet to be studied whether the population of aged stem cells might be fractionated into subgroups that bear different potency in terms of self-renewal. Such findings would impinge on any rejuvenation approach. Interestingly, the abovementioned successful rejuvenation may suggest that indeed the population of HSCs contains senescent cells [24] and their removal clear the space for the more potent ones. Recent studies using single-cell RNA-seq had discovered cell-cycle and differentiation priming clonal changes with aging [33], with further clonal bias towards myeloid- and even into platelets-restricted fate [34]. These molecular findings are in agreement with functional study of aged mouse and human HSCs [35].

HSCs do age, and their long-term potential as stem cells seems to be hindered both after intrinsic and extrinsic physiological processes. While the interest in rejuvenation has long history, recent discoveries show it is possible not only to improve immune-activity at the effector cell level, but more deeply to change the state of our HSCs pool and benefit the improved hematopoietic and immune system for prolonged time. With better understanding we might be able to carefully manipulate endogenous stem cell's population and rejuvenate HSCs clones.

\section{References}

1. Effros RB (2007) Role of $T$ lymphocyte replicative senescence in vaccine efficacy. Vaccine 25: 599-604. 
2. Gazit R, Weissman IL, Rossi DJ (2008) Hematopoietic stem cells and the aging hematopoietic system. Semin Hematol 45: 218-224.

3. Vu T, Farish S, Jenkins M, Kelly H (2002) A meta-analysis of effectiveness of influenza vaccine in persons aged 65 years and over living in the community. Vaccine 20: 1831-1836.

4. Yung RL, Julius A (2008) Epigenetics, aging and autoimmunity. Autoimmunity 41: 329-335.

5. Busch K, Klapproth K, Barile M, Flossdorf M, Holland-Letz T, et al. (2015) Fundamental properties of unperturbed haematopoiesis from stem cells in vivo. Nature 518: 542-546

6. Abramovich C, Humphries RK (2005) Hox regulation of normal and leukemic hematopoietic stem cells. Curr Opin Hematol 12: 210-216.

7. Zhang J, Niu C, Ye L, Huang $\mathrm{H}, \mathrm{He} X$, et al. (2003) Identification of the haematopoietic stem cell niche and control of the niche size. Nature 425: 836841.

8. Harrison DE, Astle CM (1982) Loss of stem cell repopulating ability upon transplantation. Effects of donor age, cell number and transplantation procedure. J Exp Med 156: 1767-1779.

9. Sieburg HB, Rezner BD, Muller-Sieburg CE (2011) Predicting clonal selfrenewal and extinction of hematopoietic stem cells. Proc Natl Acad Sci U S A 108: $4370-4375$.

10. Tümpel S, Rudolph KL (2014) Aging stem cells: Transcriptome meets epigenome meets methylome. Cell Stem Cell 14: 551-552.

11. Muszkat M, Greenbaum E, Ben-Yehuda A, Oster M, Yeu'I E, et al. (2003) Local and systemic immune response in nursing-home elderly following intranasal or intramuscular immunization with inactivated influenza vaccine. Vaccine 21 1180-1186.

12. Rossi DJ, Bryder D, Zahn JM, Ahlenius H, Sonu R, et al. (2005) Cell intrinsic alterations underlie hematopoietic stem cell aging. Proc Natl Acad Sci U S A 102: $9194-9199$

13. Waterstrat A, Van Zant G (2009) Effects of aging on hematopoietic stem and progenitor cells. Curr Opin Immunol 21: 408-413.

14. Sudo K, Ema H, Morita Y, Nakauchi H (2009) Age-associated characteristics of murine hematopoietic stem cells. J Exp Med 192: 1273-1280.

15. Beerman I, Rossi DJ (2015) Epigenetic control of stem cell potential during homeostasis, aging and disease. Cell Stem Cell 16: 613-625.

16. Linton PJ, Dorshkind K (2004) Age-related changes in lymphocyte development and function. Nat Immunol 5: 133-139.

17. Min H, Montecino-Rodriguez E, Dorshkind K (2004) Reduction in the developmental potential of intrathymic T cell progenitors with age. J Immunol 173: $245-250$.

18. You J, Dong H, Mann ER, Knight SC, Yaqoob P (2013) Ageing impairs the T cell response to dendritic cells. Immunobiology 218: 1077-1084.

19. Dunn-Walters DK, Banerjee M, Mehr R (2003) Effects of age on antibody affinity maturation. Biochem Soc Trans 31: 447-448.
20. Agarwal S, Busse PJ (2010) Innate and adaptive immunosenescence. Ann Allergy Asthma Immunol 104: 183-190.

21. Smith DL, Jr., Mattison JA, Desmond RA, Gardner JP, Kimura M, et al. (2011) Telomere dynamics in rhesus monkeys: No apparent effect of caloric restriction. J Gerontol A Biol Sci Med Sci 66: 1163-1168.

22. Cheng CW, Adams GB, Perin L, Wei M, Zhou X, et al. (2014) Prolonged fasting reduces IGF-1/PKA to promote hematopoietic-stem-cell-based regeneration and reverse immunosuppression. Cell Stem Cell 14: 810-823.

23. Geiger H, Zheng $Y$ (2014) Regulation of hematopoietic stem cell aging by the small RhoGTPase Cdc42. Exp Cell Res 329: 214-219.

24. Chang J, Wang Y, Shao L, Laberge RM, Demaria M, Campisi J, et al. (2016) Clearance of senescent cells by ABT263 rejuvenates aged hematopoietic stem cells in mice. Nat Med 22: 78-83.

25. Cheshier SH, Prohaska SS, Weissman LL (2007) The effect of bleeding on hematopoietic stem cell cycling and self-renewal. Stem Cells Dev 16: 707-717.

26. Keren Z, Averbuch D, Shahaf G, Zisman-Rozen S, Golan K, et al. (2011) Chronic $B$ cell deficiency from birth prevents age-related alterations in the $B$ lineage. J Immunol 187: 2140-2147.

27. Keren Z, Naor S, Nussbaum S, Golan K, Itkin T, et al. (2011) B-cell depletion reactivates $B$ lymphopoiesis in the $B M$ and rejuvenates the $B$ lineage in aging Blood 117: 3104-3112.

28. Cho RH, Sieburg HB, Muller-Sieburg CE (2008) A new mechanism for the aging of hematopoietic stem cells: Aging changes the clonal composition of the stem cell compartment but not individual stem cells. Blood 111: 5553-5561.

29. Beerman I, Bhattacharya D, Zandi S, Sigvardsson M, Weissman IL, et al. (2010) Functionally distinct hematopoietic stem cells modulate hematopoietic lineage potential during aging by a mechanism of clonal expansion. Proc Nat Acad Sci U S A 107: 5465-5470.

30. Challen GA, Boles NC, Chambers SM, Goodell MA (2010) Distinct hematopoietic stem cell subtypes are differentially regulated by TGF-beta1. Cell Stem Cell 6: 265-278.

31. Morita Y, Ema H, Nakauchi $H$ (2010) Heterogeneity and hierarchy within the most primitive hematopoietic stem cell compartment. J Exp Med 207: 11731182

32. Wahlestedt M, Norddahl GL, Sten G, Ugale A Frisk MA, et al. (2013) An epigenetic component of hematopoietic stem cell aging amenable to reprogramming into a young state. Blood 121: 4257-4264.

33. Kowalczyk MS, Tirosh I, HeckI D, Rao TN, Dixit A, et al. (2015) Single-cell RNAseq reveals changes in cell cycle and differentiation programs upon aging of hematopoietic stem cells. Genome Res 25: 1860-1872.

34. Grover A, Sanjuan-Pla A, Thongjuea S, Carrelha J, Giustacchini A, et al. (2016) Single-cell RNA sequencing reveals molecular and functional platelet bias of aged haematopoietic stem cells. Nat Commun 7: 11075.

35. Rundberg Nilsson A, Soneji S, Adolfsson S, Bryder D, Pronk CJ (2016) Human and murine hematopoietic stem cell aging is associated with functional impairments and intrinsic megakaryocytic/erythroid bias. PLoS One 11 e0158369. 